\title{
An Incomplete Block Change-Over Design Balanced for First and Second-Order Residual Effect
}

\author{
Kanchan Chowdhury \\ Professor, Department of Statistics, Jahangirnagar University \\ Savar, Dhaka-1342, Bangladesh \\ Rumana Rois (Corresponding author) \\ Department of Statistics, Jahangirnagar University \\ Savar, Dhaka-1342, Bangladesh \\ E-mail: rumana248@yahoo.com
}

Received: January 11, 2013 Accepted: January 24, 2013 Published: February 6, 2013

doi:10.5296/jas.v1i1.3028ＵRL: http://dx.doi.org/10.5296/jas.v1i1.3028

\begin{abstract}
Balanced designs are often needed in agriculture, economics and other context. A series of balanced designs called incomplete block change-over design (IBCOD) has been developed. The analysis and the problems of IBCOD which also provide estimates of first-order and second-order residual effects have also been presented.
\end{abstract}

Keywords: Balanced Design, Incomplete Block Change-Over Design, Residual effects.

\section{Introduction}

The experiments in which each experimental unit receives a cyclical sequence of some or all of the treatments one at a time over a certain period of time are known as change-over designs. These designs have been used advantageously in several fields of research, notably in nutrition experiments with dairy cattle, clinical trials in medical research, psychological experiments, long-term agricultural field experiments and bio-assays. These designs are widely used when homogeneous experimental units are scarce. The distinguishing feature of such an experiment is that any treatment applied to a unit not only in the period of its application but also leaves residual effects in the succeeding periods (Sharma et al., 2007). They are generally capable of providing treatments comparisons of high precision because they eliminate the difference among experimental units from the error variation.

A large volume of literature in design of experiments is available to study the change-over 
designs. These designs have been devised by several research workers: Williams (1949, 1950), Patterson (1950), Patterson and Lucas (1962), Barenblut (1964), Saha (1970), Lawless (1971), Sharma (1975), Kershner and Federer (1981), Pigeon and Raghavarao (1987), Chowdhury (1987) and Varghese et al. (2000) etc. are few of them. Literatures on higher order residual effects in the case of change-over design are limited. Hence, we are interested to construct a change-over design for first-order as well as second-order residual effects.

There are several situations where change-over designs are essential: (i) each experimental unit can be used for several tests to reduce the cost of the experiments; (ii) in most of the experiments the treatment effects do not have a serious bad effect on the experimental units and hence these units can be used for further experiments; (iii) in some experiments human beings or animals are used as experimental units and hence it needs long-term training. So, after completion of the experiments due to time constraint, some experimenters have to use these experimental units for further several tests; (iv) when the objective of the experiments is to find out the effect of different fertilizers, drugs, nutrition etc.; and (v) sometimes the experimental units are scarce therefore the experimental units have to be used repeatedly.

The organization of the paper is as follows. In section 2, we discuss the residual effects. Conditions for balanced design are discussed in section 3. We also explain the construction of incomplete block change-over design (IBCOD) in section 4. Section 5 illustrates IBCOD with an example. Analysis of our proposed IBCOD is discussed in section 6. Finally, section 7 contains concluding remarks.

\section{Residual Effects}

Change-over designs are the most used designs where treatments are applied in sequences to each experimental unit at times where the effects of certain treatments continue-during the subsequent periods get entangled with the carry over or residual effect of the previous treatment. The residual effects that persist up to the $k$-th period are known as $k$-th order residual effect. This feature of residual is the distinguishing feature of change-over designs.

Residual effects are independent of the treatments applied in the period in which they are observed. In this case direct and residual effects are said to be additive. For example, 1, 2, 3 : $2,1,3$ the measurement of the first order residual effect of treatment 1 is made in the second period of first sequence and the third period of second sequence. If $d$ is the direct effect of a treatment and $r$ the first-order residual effect, $r^{\prime}$, the second-order residual effect and so on, then the measurements in the two sequences are $d_{1}, d_{2}+r_{1}, d_{3}+r_{2}+r_{1}: d_{2}, d_{1}+r_{2}$, $d_{3}+r_{1}+r_{2}$.

\section{Condition for Balanced Design}

Direct and residual effects of treatments can be estimated without much involvement, the designs should be balanced. Designs which posses the following properties will be balanced:

- Differences of direct effects of any two treatments are estimated with equal precision.

- Differences of residual (first order and second-order) effects of two treatments are estimated with equal precision. 
Let us consider the layout plan of the design consisting of $b$ sequences for $v$ treatments in the periods such that

(i) no treatment symbol occurs in a given sequence more than once;

(ii) each treatment symbol occurs in a given period an equal number of times;

(iii) every two treatment symbols should occur together in the same number of sequences;

(iv) each ordered succession of two treatment symbols should occur equally often in the sequences;

(v) every two treatment symbols occur together in the same number of curtailed sequences formed by omitting the final period;

(vi) in those sequences where a given treatment occurs in the final period, the other treatments should also occur equally often;

(vii) in those sequences where a given treatment occur in any but the final period, each other treatment should occur equally often in the final period;

(viii) each treatment is preceded two periods earlier by each other treatment equally often;

(ix) each ordered pair of treatments occurs at the end of a sequence with equal frequencies;

(x) in those sequences in which a given treatment occurs in the last but one period the other treatments occur equally often;

(xi) in those sequences in which a given treatment occurs in any but the last but one period the other treatments occur equally often in the last but one period.

The design satisfying the conditions mentioned above will be balanced for first and second-order residual effects.

These designs are capable of providing treatments comparison of high precision because they eliminate the difference among experimental units from the error variation.

\section{Construction}

Let us consider the balance incomplete block design (BIBD) with $b=v$, $r=k=v-1, \lambda=v-2$, where $v$ is any odd number. Associating William's Special Latin Squares with each block of the above mentioned design, an incomplete block change-over design is obtained with $v=b, k=v-1, p=v-1$ which will satisfy all the conditions for balanced designs. Further instead of taking $(\mathrm{v}-1)$ periods we consider only first $p$ periods, when $p \geq 3$, then also we obtain an IBCOD design with parameters $v=b, k=v-1, p=v-1$ satisfy the conditions for balanced design of first-order and second-order residual effects. 


\section{MlMacrothink}

\section{An Illustration}

Let $\mathrm{v}=5$ be the number of treatments. A balanced incomplete block design for 5 treatments in block size four is considered.

$$
\begin{array}{llll}
1 & 2 & 3 & 4 \\
2 & 3 & 4 & 5 \\
3 & 4 & 5 & 1 \\
4 & 5 & 1 & 2 \\
5 & 1 & 2 & 3
\end{array}
$$

\begin{tabular}{|c|c|c|c|c|c|c|c|c|c|c|c|c|c|c|c|c|c|c|c|c|c|c|c|c|}
\hline \multirow{2}{*}{$\frac{\text { Period }}{1}$} & \multicolumn{4}{|c|}{ Block I } & \multicolumn{2}{|l|}{ Period } & \multicolumn{3}{|c|}{ Block II } & \multicolumn{2}{|l|}{ Period } & \multicolumn{3}{|c|}{ Block III } & \multicolumn{2}{|l|}{ Period } & \multicolumn{3}{|c|}{ Block IV } & \multicolumn{2}{|l|}{ Period } & \multicolumn{3}{|c|}{ Block V } \\
\hline & 1 & 2 & 3 & 4 & 1 & 2 & 3 & 4 & 5 & 1 & 3 & 4 & 5 & 1 & 1 & 4 & 5 & 1 & 2 & 1 & 5 & 1 & 2 & 3 \\
\hline 2 & 2 & 4 & 1 & 3 & 2 & 3 & 5 & 2 & 4 & 2 & 4 & 1 & 3 & 5 & 2 & 5 & 2 & 4 & 1 & 2 & 1 & 3 & 5 & 2 \\
\hline 3 & 3 & 1 & 4 & 2 & 3 & 4 & 2 & 5 & 3 & 3 & 5 & 3 & 1 & 4 & 3 & 1 & 4 & 2 & 5 & 3 & 2 & 5 & 3 & 1 \\
\hline 4 & 4 & 3 & 2 & 1 & 4 & 5 & 4 & 3 & 2 & 4 & 1 & 5 & 4 & 3 & 4 & 2 & 1 & 5 & 4 & 4 & 3 & 2 & 1 & 5 \\
\hline
\end{tabular}

Associating (5.1) with William's Special Latin Squares we obtain an IBCOD design with $v=b=5, k=4, p=4$.

Table 1. Sequences of IBCOD

In this above design if the last period is omitted we obtain another IBCOD design with $v=b=5, k=3, p=3$.

\section{Analysis of IBCOD Design}

Let us consider an IBCOD with parameters $v=t, b, k$ and $p$, i.e., $t$ treatments in $b$ blocks of $k$ units each and in $p$ periods. We assume the following usual fixed effects model for analysis:

$$
Y_{i j k l m n}=\mu+\pi_{i}+\beta_{j}+\alpha_{k j}+\tau_{1}+\rho_{m}+\rho_{n}^{\prime}+(\pi \beta)_{i j}+\varepsilon_{i j k l m}
$$

$i=1,2, \ldots, p$ (number of periods); $j=1,2, \ldots, b$ (number of blocks); $k=1,2, \ldots, k$ (number of units in a block); $l, m, n=1,2, \ldots, t$ (number of treatments with the restrictions).

$$
\begin{gathered}
\sum_{i} \pi_{i}=\sum_{j} \beta_{j}=\sum_{k} \alpha_{k j}(\text { for every } j)=\sum_{1} \tau_{1}=\sum_{m} \rho_{m}=\sum_{n} \rho_{n}^{\prime} \\
=\sum_{i}(\pi \beta)_{i j}=\sum_{j}(\pi \beta)_{i j}=0 .
\end{gathered}
$$




\section{Macrothink}

where, $Y_{i j k l m n}$ is the observation corresponding to the $i$-th period and $k$-th unit in the $j$-th block receiving treatment $l$ immediately preceded by treatment $m$ and immediately preceded two periods back by treatment $n$. $\mu$ denotes the general mean effect, $\pi_{i}$, $i$-th period effect, $\beta_{j}$, j-th block effect $\alpha_{k j}$, effect of the $k$-th experimental unit within the $j$-th block $\tau_{1}$ direct effect of the treatment $l, \rho_{m}$ and $\rho_{n}^{\prime}$, first and second-order residual effect of the treatment $\mathrm{m}$ and $\mathrm{n}$ respectively. $(\pi \beta)_{i j}$, the interaction effect between $i$-th period and $j$-th block and $\varepsilon_{i j k l m n}$ is the random error component assumed to be normally distributed with mean zero and constant variance $\sigma^{2}$.

Let the letters $t_{1}^{\prime} s, r_{i}^{\prime} s, r_{i}^{\prime \prime} s$ be used for the least-squares estimates of $\tau_{i}^{\prime} s, \rho_{i}^{\prime} s$ and $\rho_{i}^{\prime \prime} s$ respectively.

$i=1,2, \ldots, t$. Let us further define

(i) Sum of the block totals taking only those blocks which contain treatment i, by $\sum_{j} B_{j}^{(i)}$

(ii) Sum of the unit (sequences) totals taking only those units which contain treatment $\mathrm{i}$ (a) in the last period by $\sum_{1} S_{1}^{[i]}$, (b) in the last and last but one period together by $\sum_{1} S_{1}^{\{i\}}$,

(iii) Sum of the period totals taking those blocks which contain treatment i, (a) in the first period by $\sum_{j}(P B)_{1 j}^{(i)}$ and (b) in the first $(i=1,2, j=1,2, \ldots, b)$ and second period by $\sum_{i=1,2, j=1,2, \ldots, b}(P B)_{i j}^{(i)}$.

Now it can be shown that for an IBCOD design, the reduced normal equations for estimating direct and first and second-order residual effect under the model (6.1) come out as follows:

$$
\begin{aligned}
& T_{i}=r k m+k \sum_{j \neq i} b_{j}^{(i)}+r k t_{i}+\lambda \sum_{j \neq i} r_{j}+(\lambda-1) \sum_{j \neq i} r_{j}^{\prime} ; \\
& R_{i}=r(k-1) m-k P_{1}+(k-1) \sum_{j \neq i} b_{j}^{(i)}-\sum_{k j} s_{k j}+\lambda \sum_{j \neq i} t_{j}+r(k-1) r_{i}-\sum_{j}\left(p \phi_{1 j}+(\lambda-1) \sum_{j \neq i} r_{j}^{\prime} ;\right. \\
& R_{i}^{\prime}=r(k-2) m-k\left(P_{1}+P_{2}\right)+(k-2) \sum_{j} b_{j}^{(i)}-\sum_{k j} s_{k j}
\end{aligned}
$$$$
\text { (for first two periods) }
$$ 


$$
+(\lambda-1) \sum_{j \neq i} t_{j}+(\lambda-1) \sum_{j \neq i} r_{j}+r(k-2) r_{i}^{\prime}-\sum_{\substack{i=1,2 \\ j=1,2, \ldots b}}(p b)_{i j}
$$

$$
\begin{aligned}
\sum_{j} B_{j}^{(i)}=r k^{2} m & +k^{2} \sum_{j} b_{j}^{(i)}+r k t_{i}+\lambda k \sum_{j \neq i} t_{j}+r(k-1) r_{i} \\
& +\lambda(k-1) \sum_{j \neq i} r_{j}+r(k-2) r_{i}^{\prime}+\lambda(k-2) \sum_{j \neq i} r_{j}^{\prime} ;
\end{aligned}
$$

$$
\sum_{1} s_{1}^{[i]}=r k m+k \sum_{j} b_{j}^{[i]}+k \sum_{k j} s_{k j}+k t_{i}+\lambda \sum_{j \neq i} t_{j}+\lambda \sum_{j \neq i} r_{j}+(\lambda-1) \sum_{j \neq i} r_{j}^{\prime}
$$

$$
\begin{aligned}
\sum_{1} s_{1}^{\{i\}}=2 r k m & +2 k \sum_{j} b_{j}^{\{i\}}+k \sum_{k, j} s_{k j}+2 k t_{i}+2 \lambda \sum_{j \neq i} t_{j}+k r_{i} \\
& +(2 \lambda-1) \sum_{j \neq i} r_{j}+(2 \lambda-2) \sum_{j \neq i} r_{j}^{\prime}
\end{aligned}
$$

$$
\begin{gathered}
\sum_{j}(P B)_{1 j}=r k m+k^{2} p_{1}+k \sum_{j} b_{j}^{(i)}+k t_{i}+\lambda \sum_{j \neq i} t_{j}+k r_{i}+k \sum_{j}(p b)_{i j} \\
{\left[\sum_{j}(P B)_{1 j}+\sum_{j}\left(P B_{2 j}\right)\right]=2 r k m+k^{2}\left(P_{1}+P_{2}\right)+2 k \sum_{j} b_{j}^{(i)}+2 k t_{i}} \\
+2 \lambda \sum_{j \neq i} t_{j}+k r_{i}+\lambda \sum_{j \neq i} r_{j}+k \sum_{\substack{i=1,2 \\
j=1,2, \ldots b}}(p b)_{i j}
\end{gathered}
$$

where, $T_{i}$ is the total of the observations from the plots having treatments $i, R_{i}$, the totals of the observations from the plots which had treatment $i$ in the preceding period and $R_{i}^{\prime}$ the totals of the observations from the plots which had treatment $i$ in preceding two periods.

Eliminating block effect, sequence effect, period effect, the reduced normal equations are:

$$
\begin{gathered}
P_{i}=c\{r(k-1)+\lambda\} t_{i}-c\{[r(k-1)+\lambda] / k\} r_{i}-c\{[r(k-3)+2 \lambda] / k\} r_{i}^{\prime} ; \\
Q_{i}=-c\{[r(k-1)+\lambda] / k\} t_{i}+\left[c\left(k^{2}-k-1\right) / k^{2}\right]\{r(k-1)+\lambda\} r_{i} \\
-c\left\{\left[\left(k^{2}-k-2\right)(r-\lambda)+k(\lambda-1)(k+1)\right] / k^{2}\right\} r_{i}^{\prime}
\end{gathered}
$$




$$
\begin{aligned}
M_{i}=-c & \{r[(k-3)+2 \lambda] / k\} t_{i}-c\left\{\left[\left(k^{2}-k-2\right)(r-\lambda)+k(\lambda-1)(k+1)\right] / k^{2}\right\} r_{i} \\
& +c\left\{\left[r(k-2)\left(k^{2}-k-2\right)+\lambda\left(k^{2}-4\right)-2 k(\lambda-2)\right] k^{2}\right\} r_{i}^{\prime}
\end{aligned}
$$

where, $P_{i}=T_{i}-(1 / k) \sum_{j} B_{j}^{(i)}$,

$$
Q_{i}=R_{i}-\left[(k+1) / k^{2}\right] \sum_{j} B_{j}^{(i)}+\left(\frac{1}{k}\right) \sum_{1} s_{1}^{[i]}+\left(\frac{1}{k}\right) \sum_{j}(P B)_{1 j}
$$

and

$$
M_{i}=R_{i}^{\prime}-\left[(k+2) / k^{2}\right] \sum_{j} B_{j}^{(i)}+\frac{1}{k} \sum_{1} s_{1}^{\{i\}}+\frac{1}{k}\left[\sum_{j}(P B)_{1 j}+\sum(P B)_{2 j}\right]
$$

and $\mathrm{c}=1$, when $\mathrm{v}$ is even and $\mathrm{c}=2$, when $v$ is odd.

On simplifications, assuming $\mathrm{c}=1$ here, the estimates of direct, first and second-order residual effect are as follows:

$$
\begin{aligned}
& \hat{t}_{i}=[A / \Delta] p_{i}+[B / \Delta] Q_{i}+[C / \Delta] M_{i} \\
& \hat{r}_{i}=[B / \Delta] p_{i}+[D / \Delta] Q_{i}+[E / \Delta] M_{i} ; \\
& \hat{r}_{i}^{\prime}=[C / \Delta] p_{i}+[E / \Delta] Q_{i}+[F / \Delta] M_{i}
\end{aligned}
$$

where,

$$
\begin{aligned}
A= & \left(1 / k^{4}\right)\{r(k-1)+\lambda\}\left(k^{2}-k-1\right)\left[r(k-2)\left(k^{2}-k-2\right)+\lambda\left(k^{2}-4\right)-2 k(\lambda-1)\right] \\
& -\left(1 / k^{4}\right)\left[\left(k^{2}-k-2\right)(r-\lambda)+k(\lambda-1)(k+1)\right]^{2} ; \\
B=\left\{[r(k-1)+\lambda] / k^{3}\right\}\left\{r(k-2)\left(k^{2}-k-2\right)+\lambda\left(k^{2}-4\right)-2 k(\lambda-1)\right\} & \\
& +\left[(r k-3 r+2 \lambda) / k^{3}\right]\left\{\left(k^{2}-k-2\right)(r-\lambda)+k(\lambda-1)(k+1)\right\} ; \\
C= & \left\{[r(k-1)+\lambda] / k^{3}\right\}\left\{\left(k^{2}-k-2\right)(r-\lambda)+k(\lambda-1)(k+1)\right\} \\
+ & {\left[(r k-3 r+2 \lambda) / k^{3}\right]\{r(k-1)+\lambda\}\left(k^{2}-k-1\right) ; } \\
D= & \left\{[r(k-1)+\lambda] / k^{2}\right\}\left\{r(k-2)\left(k^{2}-k-2\right)+\lambda\left(k^{2}-4\right)-2 k(\lambda-1)\right\} \\
& -\left\{[r(k-3)+2 \lambda] / k^{4}\right\}^{2} ;
\end{aligned}
$$




$$
\begin{aligned}
& \begin{array}{c}
E=\left\{[r(k-1)+\lambda] / k^{2}\right\}\left\{\left(k^{2}-k-2\right)(r-\lambda)+k(\lambda-1)(k+1)\right\} \\
+[\{r(k-1)+\lambda\}\{r(k-3)+2 \lambda\}] / k^{2} ;
\end{array} \\
& F=\left\{[r(k-1)+\lambda] / k^{2}\right\}^{2}\left(k^{2}-k-1\right)-\left\{[r(k-1)+\lambda] / k^{2}\right\}^{2} ; \\
& \text { and } \Delta=\{r(k-1)+\lambda\} A-\{[r(k-1)+\lambda] / k\} B-\{[r(k-3)+2 \lambda] / k\}
\end{aligned}
$$

From (6.3) the estimates of variances are as follows:

$$
\operatorname{var}\left(t_{i}-t_{j}\right)=2 A \sigma^{2} / \Delta, \operatorname{var}\left(r_{i}-r_{j}\right)=2 D \sigma^{2} / \Delta \operatorname{var}\left(r_{i}^{\prime}-r_{j}^{\prime}\right)=2 F \sigma^{2} / \Delta \quad i \neq j=1,2, \ldots, t
$$

Following Lucas and Patterson (1962), the efficiency factors for direct, first and second-order residual effects respectively are as follows:

$$
\begin{aligned}
& E_{d}=\left[2 \sigma^{2} / n k\right] / \operatorname{var}\left(t_{i}-t_{j}\right)=\Delta / \text { Ank } \\
& E_{r}=\left[2 \sigma^{2} / n k\right] / \operatorname{var}\left(r_{i}-r_{j}\right)=\Delta / \text { Dnk } \\
& E_{s r}=\left[2 \sigma^{2} / n k\right] / \operatorname{var}\left(r_{i}^{\prime}-r_{j}^{\prime}\right)=\Delta / \text { Fnk }
\end{aligned}
$$

Table 2. Analysis of Variance of IBCOD

\begin{tabular}{cccc}
\hline Variation due to & Degrees of Freedom & S.S & M.S.S \\
\hline Blocks & $(\mathrm{b}-1)$ & + & + \\
Periods & $(\mathrm{p}-1)$ & + & + \\
Block x Periods & $(\mathrm{b}-1)(\mathrm{p}-1)$ & $\sum_{i=1} t_{i} p_{i}$ & \\
Units within block & $\mathrm{b}(\mathrm{k}-1)$ & $\sum_{i} r_{i} Q_{i}$ & \\
Direct Effect & $(\mathrm{v}-1)$ & $\sum_{i} r_{i}^{\prime} M_{i}$ & $S_{e}^{2}$ \\
First order residual & $(\mathrm{v}-1)$ & & $s_{e}^{2 *}=\frac{\text { error } d . f .}{\text { effect }}$ \\
$\begin{array}{c}\text { Second-order } \\
\text { residual effect }\end{array}$ & $(\mathrm{v}-1)$ & $S_{e}^{2}$ & $(\mathrm{by}$ subtraction $)$ \\
Error & $\mathrm{NP}-\mathrm{b}(\mathrm{p}+\mathrm{k}-1)-3$ & & \\
Total & $(\mathrm{v}-1)$ & $\mathrm{NP}-1$ &
\end{tabular}

+ Calculated in the usual way,

$* s_{e}^{2}$ is an estimate of $\sigma^{2}$

$\mathrm{N}$ is the total number of units (sequences) in the design. 


\section{Conclusion}

Change-over designs are generally proposed to get direct and first-order residual effects of treatments when treatments are applied in sequences. In this study this problem has been explored with the assumptions of presence of second-order residual effects. In some practical situations, in particular in triple cropping system which is prevalent in Bangladesh the second-order residual effect cannot be ignored. So, the investigation made in this study would be useful for agricultural country like Bangladesh.

\section{References}

Barenblut, I. I. (1964). Change-over designs with Complete Balanced for first Order Residual Effects, Biometrics, 20, 707-712.

Chowdhury, K. (1987). Some Contribution to Construction and analysis of Incomplete Factorial Experiments, An Unpublished Ph.D thesis, Department of Agricultural Statistics, Bidhan Chandra Krishi Viswavidyalaya, India.

Kershner, R. P., \& Federer, W. T. (1981). Two-treatment crossover designs for estimating a variety of effects. J. Amer. Statist. Assoc., 76 (375), 612-619. http://dx.doi.org/10.2307/2287519

Lawless, J. F. (1971). A note on certain types of BIBDs balanced for first residual effects. Ann. Math. Statist., 42(4), 1439-1441. http://dx.doi.org/10.1214/aoms/1177693256

Patterson, H. D. (1950). The Analysis of Change-over Trials, Journal of Agricultural Science, 40, 375-380. http://dx.doi.org/10.1017/S002185960004613X

Patterson, H. D., \& Lucas, H. L. (1962). Change-over Designs, North Carolina Agri. Expt. Sta. Tech. Bull., No. 147.

Pigeon, J. G., \& Raghavarao, D. (1987). Crossover designs for comparing treatments with a control. Biometrika, 74 (2), 321-328. http://dx.doi.org/10.1093/biomet/74.2.321

Saha, G. M. (1970). Some Contributions to Design and Analysis of Experiments Involving Sequences of Treatments, Unpublished Ph.D thesis, Institute of Agricultural Research Statistics, New Delhi.

Sharma, A., Varghese, C., Rao, A. R., Gupta, V. K., \& Pal, S. (2007). SPRMD—A Statistical Package for Cataloguing and Generation of Repeated Measurement Designs. J Ind. Soc. Agril. Statist, 61(1), 84-90.

Sharma, V. K. (1975). An Easy Method of Constructing Latin Square Design Balanced for the Immediate Residual and Other Order Effects. Canadian J. Stat., 3, 119-124. http://dx.doi.org/10.2307/3315104

Varghese, C., Vijaya, B., \& Sharma, V. K. (2000). Cross-over designs for comparison of two treatments in presence of residual effects. Ind. J. Anim. Sci., 70(8), 862-865.

Williams, E. J. (1949). Experimental Designs Balanced for the Estimation of Residual Effects 


\section{Macrothink

of treatments. Austral. Jour. Sci. Res. Ser. A, 2, 149. http://dx.doi.org/10.1071/CH9490149

Williams, E. J. (1950). Experimental Designs Balanced for Pairs of Residual Effects. Austral. Jour. Sci. Res. Ser. A, 3, 351. http://dx.doi.org/10.1071/CH9500351

\section{Copyright Disclaimer}

Copyright reserved by the author(s).

This article is an open-access article distributed under the terms and conditions of the Creative Commons Attribution license (http://creativecommons.org/licenses/by/3.0/). 